\title{
Projeção da distribuição diamétrica de uma floresta explorada seletivamente na Amazônia Ocidental
}

\author{
Sumaia S. VASCONCELOS ${ }^{1}$, Niro HIGUCHI ${ }^{2}$, Marcus V.N. OLIVEIRA ${ }^{3}$ \\ RESUMO \\ Utilizando um modelo estocástico, foi projetada a distribuição diamétrica futura de uma floresta submetida à exploração seletiva \\ de madeira na Amazônia Ocidental. Foram utilizados dados de cinco parcelas permanentes localizadas no PC Pedro Peixoto, no \\ Acre. A primeira medição das parcelas ocorreu em 1996, a exploração florestal em 1997 e as re-medições em 1999 e 2001. A \\ principal variável utilizada foi o diâmetro à altura do peito (DAP). A matriz de transição probabilística (Cadeia de Markov) foi \\ utilizada para fazer a projeção da distribuição diamétrica do número de árvores sobreviventes nas classes diamétricas. O modelo \\ foi primeiramente testado para fazer a projeção para 2001, tendo como base as observações de 1999 e seu passado imediato \\ (1997). Quando comparadas às projeções feitas para 2001 e as mediçóes de campo (2001), o teste Qui-quadrado mostrou \\ que não houve diferença significativa entre freqüências esperadas e observadas na distribuição diamétrica $(p=0,05)$. A projeção \\ para 2005 indica que a taxa de mortalidade será próxima a de 2001, e se repetida a taxa de recrutamento em 2005 o total de \\ árvores será maior que o observado em 2001. Esse comportamento da floresta indica que não existe um padrão definido para \\ a dinâmica nas classes diamétricas em termos de mortalidade ou crescimento, apresentando um comportamento aleatório ou \\ probabilístico, justificando a eficiência da Cadeia de Markov para projetar a dinâmica da floresta estudada, podendo auxiliar \\ na determinação do ciclo de corte ou mostrando as tendências que a floresta de hoje apresentará em um futuro próximo.
}

PALAVRAS-CHAVE: Exploração seletiva, Distribuição diamétrica, Cadeia de Markov, Amazônia.

\section{Projection of the diameter distribution in a selective logging forest in the western Amazon}

\begin{abstract}
The diameter distribution of an experimental forest stand in the Western Amazon was projected using a stochastic model after selective logging. The study was developed using data from five permanent plots located in the colonization project Pedro Peixoto, in the state of Acre. Initial measurements of diameter at breast height (DBH) were taken in 1996 . The forest was selectively logged in 1997 and DBHs were re-measured in two different occasions, 1999 and 2001. A probabilistic transition matrix (Markov Chain) was used to project the diameter distribution of the number of surviving trees in each diameter class. The model was first tested to project the diameter distribution in 2001, based on DBH measurements from 1997 and 1999. When the projected diameter distribution for 2001 was compared with the field data from the same year, a Chi-squared test $(\alpha=0.05)$ showed that there was not significant difference between the expected and observed diameter distribution. After that, a projection for 2005 (four years in the future) was run using DBH measurements from 1997 to 2001, indicating that mortality rate was similar to 2001 . If repeated the rate of recruitment of 2005 , the total number of trees will be higher than observed in 2001. The dynamics of the studied forest suggests that there is not a definitive pattern to changes in diameter distribution and mortality, which indicates a stochastic or probabilistic pattern. This pattern is better modeled by the Markov Chain to project the forest dynamics of studied area, and can help on determination of timber harvesting or the tendencies of forest dynamics in a near future.
\end{abstract}

KEYWORDS: Selective logging, Diameter distribution, Markov Chain, Amazon.

1 Instituto Nacional de Pesquisas da Amazônia (INPA), Coordenação de Pós-Graduação em Silvicultura Tropical, Caixa Postal 478, CEP 69.011-970, Manaus-Amazônas-Brasil. sumaiasv@yahoo.com.br, niro@inpa.gov.br

2 Empresa Brasileira de Pesquisa Agropecuária (EBRAPA), Centro de Pesquisa Agroflorestal do Acre, BR-364 km 14, CEP 69.900-000, Rio Branco-Acre-Brasil. mvno@cpafac. embrapa.br 


\section{INTRODUÇÃO}

A demanda por produtos madeireiros e não madeireiros oriundos de áreas manejadas têm aumentado consideravelmente na região Amazônica. Diante disso, muitas pesquisas têm sido realizadas visando conhecer técnicas ou alternativas que possam assegurar a sustentabilidade desses recursos em florestas tropicais em regime de rendimento sustentado. A falta de informaçōes sobre o crescimento florestal e o comportamento da estrutura diamétrica no tempo, têm sido um fator limitante para o manejador florestal. A área basal e o volume de um povoamento podem ser convenientemente calculados a partir do número de árvores em cada classe diamétrica. $\mathrm{O}$ crescimento desses parâmetros pode ser estimado pela mudança na freqüência de indivíduos que resulta na mudança do número de árvores de uma classe diamétrica para outra $\mathrm{e}$ pelas taxas de recrutamento e mortalidade (Sanquetta et al. 1996; Sanquetta et al, 1999).

Os estudos de crescimento de povoamentos florestais são baseados em modelos clássicos de produção florestal, os quais foram desenvolvidos para florestas temperadas, utilizando variáveis de difícil acesso em florestas tropicais como idade das árvores e índice de sítio, o que torna muito difícil aplicar esses modelos na floresta amazônica devido a grande variabilidade da idade, além da dificuldade em obtê-la (Rocha, 2001). Outro meio que pode ser utilizado para prognosticar a dinâmica de uma floresta é por meio de parcelas permanentes. Novamente dificuldades são encontradas pelo fato de que na floresta tropical da Amazônia brasileira existe um número reduzido de parcelas permanentes, essas não são bem distribuídas e possuem tamanhos variados (Vasconcelos, 2003).

Como alternativa para estudar e projetar o crescimento de povoamentos florestais na Amazônia foi utilizado um modelo de produção florestal, que depende de uma situação imediatamente anterior a atual para a projeção de uma situação imediatamente posterior. Até o momento a Cadeia de Markov tem sido amplamente utilizada no estudo da previsão de crescimento em florestas tropicais, visto que é o mais adequado à floresta Amazônica por ser um processo estocástico para a predição diamétrica, número de árvores mortas, número de árvores sobreviventes e número de árvores colhidas (Rocha, 2001).

Diversos estudos têm desenvolvido procedimentos para a caracterização da distribuição diamétrica futura em florestas nativas. Na Indonésia destacam-se os trabalhos de Mendoza \& Setyarso (1986) e Solomon et al. (1986); No Brasil, visando assegurar o manejo sustentado destacam-se os estudos de Higuchi (1987), Silva (1989), Higuchi \& Vieira (1990), Azevedo (1993), Freitas \& Higuchi (1993), Rocha (2001), Vasconcelos (2003) e Teixeira et al. (2007). No cerrado brasileiro, destacam-se os trabalhos de Costa Neto \& Couto (1991), Scolforo \& Silva (1993), Durigan et al. (1993) e Pulz (1998).
O objetivo geral desse estudo foi projetar a distribuição diamétrica, utilizando a Cadeia de Markov, em uma floresta tropical após exploração seletiva de madeira. Os objetivos específicos foram: (1) a partir das mediçōes do diâmetro à altura do peito (DAP) dos anos de 1997 e 1999 (intervalo de dois anos), projetar a distribuição diamétrica para 2001, utilizando a Cadeia de Markov; (2) avaliar a qualidade das projeçôes feitas para 2001, por meio do teste Qui-quadrado $\left(\chi^{2}\right)$; e (3) usando as medições de DAP de 1997 e 2001 (intervalo de quatro anos), projetar a distribuição diamétrica para 2005, utilizando a Cadeia de Markov.

\section{MATERIAL E MÉTODOS}

\section{ÁREA DE ESTUDO}

O estudo foi realizado no Projeto de Colonização Pedro Peixoto (PC - Pedro Peixoto) que abrange uma área de 317.588 ha e está localizado no Estado do Acre (Figura 1) onde a Empresa Brasileira de Pesquisa Agropecuária (Embrapa) desenvolve um projeto de Manejo Florestal Comunitário utilizando o sistema de manejo florestal sustentado de impacto reduzido (Oliveira, 2000).

O clima é do tipo Awi (Köppen) e temperatura média anual de $25^{\circ} \mathrm{C}$, com um período seco entre os meses de julho a setembro e período chuvoso entre os meses de outubro a abril (Embrapa, 1996). Os solos predominantes são Latossolos Amarelos distróficos, com alto teor de argila e Argissolos Vermelhos. A topografia predominante é plana, com declividade em torno de $5 \%$. A vegetação predominante é de floresta tropical semiperenifólia, com formação de floresta aberta e floresta densa (SEPLAN, 2000).

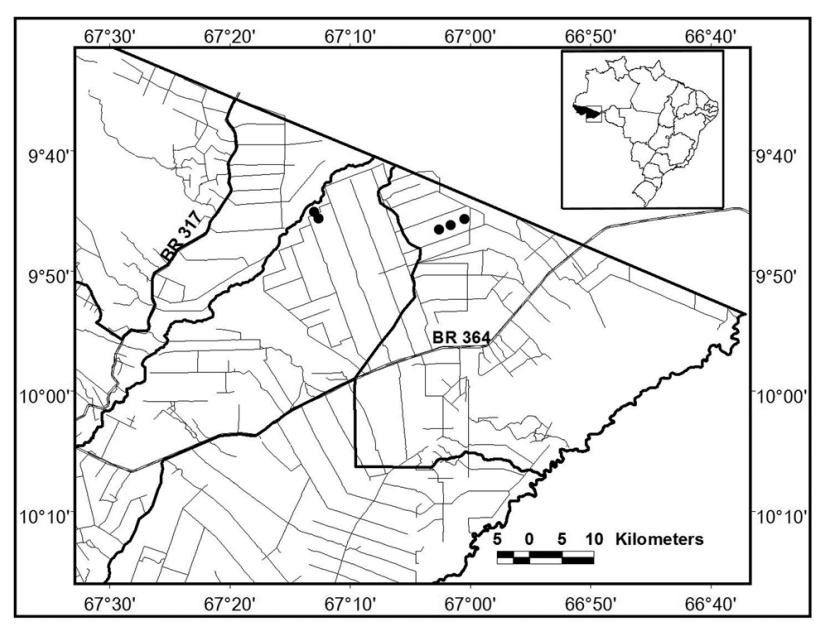

Figura 1 - Localização geográfica da área de estudo. As cinco parcelas permanentes (pontos pretos) estão estabelecidas no Projeto de Colonização Pedro Peixoto, Estado do Acre (área em destaque no mapa do Brasil) na Amazônia Ocidental. 


\section{COLETA DE DADOS}

Os dados foram coletados em cinco parcelas permanentes de um hectare cada, alocadas dentro das áreas de manejo florestal. Todas as árvores com DAP $\geq 20 \mathrm{~cm}$, localizadas dentro das parcelas permanentes, foram medidas com fita métrica, plaqueteadas com placas de alumínio e identificadas. As medições foram realizadas em três ocasióes: a primeira em 1997, após a exploração. As segunda e terceira mediçōes foram realizadas em 1999 e 2001, quando foi remedido o DAP de todos os indivíduos já registrados, bem como dos indivíduos que entraram para a primeira classe medida (ingresso), além de identificar e registrar as árvores que morreram (mortalidade) ao logo do período observado.

Os dados foram utilizados para o desenvolvimento de modelos estatísticos que descrevem o rendimento da floresta de terra firme. Esses modelos serviram de calibragem para as projeções feitas com o uso da matriz de transição probabilística.

\section{MONTAGEM DA CADEIA DE MARKOV}

Os pontos mais importantes na montagem de uma Cadeia de Markov são: a definição do espaço de estado do sistema e a construção da matriz de transição probabilística (Sanquetta et al., 1996). O espaço de estado do sistema foi composto por 16 estados: $\mathrm{a}_{1}$ (recrutamento - $\mathrm{R}$ ), $\mathrm{a}_{2} \mathrm{a}_{14}$ (classes diamétricas em intervalos de $5 \mathrm{~cm}$ ), $\mathrm{a}_{15}$ (próxima classe diamétrica) e $a_{16}$ (mortalidade - M). Para a construção de cada matriz de transição probabilística, o primeiro passo foi a construção de uma tabela contendo a freqüência absoluta das transiçôes entre os estados no intervalo de tempo considerado. A dinâmica do sistema foi representada em cada intervalo de tempo considerado da seguinte forma: mudança para classes diamétricas superiores pelo incremento diamétrico; permanência na mesma classe diamétrica (o incremento em diâmetro foi insuficiente para uma mudança de classe); mortalidade (mudança de qualquer estado para o estado $a_{16}$ ) e recrutamento (mudança do estado $a_{1}$ para qualquer um dos estados $\left.\mathrm{a}_{2} \mathrm{a}_{15}\right)$.

Após a montagem da tabela de transição foi obtida, para cada intervalo de projeçãa, a matriz de transição probabilística $\left(\mathrm{p}_{\mathrm{ij}}\right)$. As entradas dessa matriz contêm as probabilidades associadas à transição entre estados. Cada probabilidade foi calculada a partir dos elementos com as freqüências absolutas de cada estado, por meio da seguinte relação (Higuchi, 1987):

$$
\mathrm{p}_{\mathrm{ij}}=\mathrm{n}_{\mathrm{ij}} / \mathrm{N}_{\mathrm{j}}
$$

onde:

$\mathrm{p}_{\mathrm{ij}}$ - probabilidade de ocorrência de indivíduos na classe $j$, no tempo $t+1$ (segunda ocasiáo considerada), dado a classe $i$ no tempo $t$ (primeira ocasião considerada). $\mathrm{n}_{\mathrm{ij}}$ - número de indivíduos na classe $j$ no tempo $t+1$ (segunda ocasião), dado a classe $i$ no tempo $t$ (primeira ocasião considerada).

$\mathrm{N}_{\mathrm{j}}$ - número total de árvores no estado $i$ no tempo $t$.

Foram construídas matrizes de transição para os períodos de 1997/1999 e 1997/2001 (tempo $t /$ tempo $t+1$ ). De posse das matrizes de transição probabilística de cada período, a projeção para o $n$-ésimo período foi obtida pela seguinte equação (Bruner e Moser, 1973):

$$
\mathrm{p}^{(\mathrm{n})}=\mathrm{p}^{\mathrm{n}}
$$

onde:

n - número de tentativas, ou seja, a probabilidade que uma população vai de um estado $i$ de uma tentativa para o estado $j$, várias tentativas depois.

$\mathrm{p}^{(\mathrm{n})}$ - é a matriz de transição probabilística da $n$-ésima etapa.

$\mathrm{p}^{\mathrm{n}}$ - é a matriz de transição probabilística inicial elevada a $n$-ésima potência.

Com base nos dados coletados em 1997/1999 (período de dois anos), foram feitas projeçôes para o ano de 2001 (tempo $t+2$ ) e com base nos dados coletados em 1997/2001 (período de quatro anos), foram feitas projeçōes para o ano de 2005 (tempo $t+2$ ).

\section{AVALIAÇÃO DAS PROJEÇÕES PARA 2001}

Para avaliar a qualidade das projeçóes feitas para o ano de 2001 e poder dizer se o modelo proposto é bom para projetar o comportamento futuro dessa floresta, foram comparadas as frequiências projetadas e observadas de 2001 nas cinco parcelas permanentes por meio do teste $\chi^{2}$, a $5 \%$ de significância.

Nas projeçōes feitas para a distribuição diamétrica para 2001, foi realizada a correção na freqüência projetada nas classes diamétricas de $20-25 \mathrm{~cm}, 25-30 \mathrm{~cm}$ e $30-35 \mathrm{~cm}$, visando simular o recrutamento ocorrido durante cada período de projeção. Após a projeção usual feita pelo modelo markoviano, que é baseada apenas na mudança das árvores para classes diamétricas superiores e na saída do indivíduo do sistema pela mortalidade em cada classe, foi somado a essas classes, o número de árvores que seriam recrutadas no período considerado para a projeção. Foi utilizado o recrutamento médio observado para o período de 1997/1999, para cada classe, visto que as projeçôes são feitas em curto prazo.

\section{RESULTADOS E DISCUSSÃO}

\section{PROJEÇÃO PARA 0 ANO DE 2001}

Com base nas medições de 1997 e 1999, foram feitas projeçōes para o ano de 2001, com o objetivo de comparar com as medições do mesmo ano. A Tabela 1 apresenta a transição do número de árvores, do ponto de vista absoluto, 
Tabela 1 - Transição do número de árvores em cada classe diamétrica em DAP (cm), durante o período de 1997 - 1999.

\begin{tabular}{|c|c|c|c|c|c|c|c|c|c|c|c|c|c|c|c|c|c|}
\hline CD & $\mathrm{R}$ & 20 & 25 & 30 & 35 & 40 & 45 & 50 & 55 & 60 & 65 & 70 & 75 & $\geq 80$ & Próx & M & 1997 \\
\hline $\mathrm{R}$ & & 33 & 2 & 1 & & & & & & & & & & & & & 36 \\
\hline 20 & & 144 & 20 & & & & & & & & & & & & & 14 & 178 \\
\hline 25 & & & 97 & 15 & 1 & & & & & & & & & & & 13 & 126 \\
\hline 30 & & & & 58 & 7 & & & & & & & & & & & 10 & 75 \\
\hline 35 & & & & & 58 & 13 & & & & & & & & & & 2 & 73 \\
\hline 40 & & & & & & 38 & 8 & & & & & & & & & 0 & 46 \\
\hline 45 & & & & & & & 20 & 3 & & & & & & & & 3 & 26 \\
\hline 50 & & & & & & & & 18 & 3 & & & & & & & 1 & 22 \\
\hline 55 & & & & & & & & & 20 & 1 & & & & & & 3 & 24 \\
\hline 60 & & & & & & & & & & 10 & & & & & & 0 & 10 \\
\hline 65 & & & & & & & & & & & 8 & 1 & & & & 1 & 10 \\
\hline 70 & & & & & & & & & & & & 2 & 2 & & & 1 & 5 \\
\hline 75 & & & & & & & & & & & & & 1 & & & 0 & 1 \\
\hline$\geq 80$ & & & & & & & & & & & & & & 10 & 4 & 1 & 15 \\
\hline \multicolumn{18}{|l|}{ Próx } \\
\hline \multicolumn{18}{|l|}{$M$} \\
\hline 1999 & & 177 & 119 & 74 & 66 & 51 & 28 & 21 & 23 & 11 & 8 & 3 & 3 & 10 & 4 & 49 & 647 \\
\hline
\end{tabular}

$\mathrm{CD}$ - classes diamétricas; $\mathrm{R}$ - recrutamento; $\mathrm{M}$ - mortalidade.

de um estado para outro, ocorrida durante o período de 1997 1999 (intervalo de dois anos), usando todos os dados das cinco parcelas permanentes ( $5 \mathrm{ha}$ ).

O recrutamento foi de 36 árvores, isso significa que, 36 indivíduos entraram no sistema durante o período considerado para a projeção. O estado mortalidade apresentou 49 indivíduos durante o período considerado para a projeção, ou seja, 49 indivíduos saíram do sistema. Foi observado em 1997 um total de 611 árvores com DAP $\geq 20 \mathrm{~cm}$ nas cinco parcelas permanentes, 647 - 36 (árvores recrutas), e o número total de árvores observadas em 1999 foi de 598, 647 - 49 (número de árvores mortas observadas em 1999).

A Tabela 2 apresenta a matriz $\mathrm{p}_{\mathrm{ij}}$, montada a partir $\mathrm{da}$ Tabela 1, onde podem ser observadas as probabilidades, ou seja, a chance de cada árvore em cada estado, seja para permanecer na mesma classe diamétrica, mudar para uma ou mais classes imediatamente superiores ou sair do sistema por meio da mortalidade.

Em geral, as árvores têm maiores probabilidades de permanecerem na mesma classe diamétrica, principalmente considerando um período curto, de apenas dois anos. Por exemplo, a probabilidade associada à permanência de árvores da classe 20-25 cm no período de 1997 a 1999, é dada por $144 / 178=0,809$, isto é, 80,9\% do total de árvores dessa classe não apresentaram crescimento em diâmetro suficiente para uma mudança de classe no período considerado. De forma análoga, a mortalidade na mesma classe é dada por 14/178 = 0,079, ou seja, 7,9 \% das árvores dessa classe morreram no período considerado para a projeção. Dessa forma foi calculado cada elemento da matriz $\mathrm{p}_{\mathrm{ij}}$.

Para a mortalidade, foi observado que não há um padrão definido, pois as árvores pertencentes às classes diamétricas menores têm as mesmas probabilidades de morreram que as árvores das classes diamétricas maiores. Esse comportamento é típico de floresta tropical úmida o que dificulta os trabalhos de modelagem da dinâmica da floresta, principalmente quando se usam modelos determinísticos (Higuchi, 1987). Essa tendência também foi observada por Rocha (2001) e Teixeira et al. (2007) em estudos realizados em florestas naturais na Amazônia Central. No entanto, no estado 1 (recrutamento), ao contrário do estado 14 (mortalidade), 91,7\%, ocorreu na primeira classe diamétrica.

A Tabela 3 apresenta a projeção da distribuição dois passos adiante (2001), considerando a matriz $\mathrm{p}_{\mathrm{ij}}$ que foi realizada utilizando as probabilidades da matriz $\mathrm{p}_{\mathrm{ij}}{ }^{2}$.

A Tabela 3 é a Tabela 2 elevada ao quadrado, onde, por exemplo, na classe $20-25 \mathrm{~cm}$, de acordo com as probabilidades da matriz pij ${ }^{2}$ para o ano de 2001, 65,4\% das árvores existentes nessa classe no ano de 1999 não apresentarão crescimento em diâmetro suficiente para passar para a próxima classe, 17,7\% passarão para a classe $25-30 \mathrm{~cm}, 1,3 \%$ passarão para a classe $30-35 \mathrm{~cm}$ e $0,1 \%$ passarão para a classe $35-40 \mathrm{~cm}$. 
ACTA

AMAZONICA

Tabela 2 - Transição probabilística de um estado (i) para outro (j), durante um período de dois anos após a exploração (1997 - 1999).

\begin{tabular}{|c|c|c|c|c|c|c|c|c|c|c|c|c|c|c|c|c|}
\hline$C D$ & $\mathrm{R}$ & 20 & 25 & 30 & 35 & 40 & 45 & 50 & 55 & 60 & 65 & 70 & 75 & $\geq 80$ & Próx & $M$ \\
\hline $\mathrm{R}$ & & 0,917 & 0,056 & 0,028 & & & & & & & & & & & & 0 \\
\hline 20 & & 0,809 & 0,112 & & & & & & & & & & & & & 0,079 \\
\hline 25 & & & 0,770 & 0,119 & 0,008 & & & & & & & & & & & 0,103 \\
\hline 30 & & & & 0,773 & 0,093 & & & & & & & & & & & 0,133 \\
\hline 35 & & & & & 0,795 & 0,178 & & & & & & & & & & 0,027 \\
\hline 40 & & & & & & 0,826 & 0,174 & & & & & & & & & 0 \\
\hline 45 & & & & & & & 0,769 & 0,115 & & & & & & & & 0,115 \\
\hline 50 & & & & & & & & 0,818 & 0,136 & & & & & & & 0,045 \\
\hline 55 & & & & & & & & & 0,833 & 0,042 & & & & & & 0,125 \\
\hline 60 & & & & & & & & & & 1,000 & & & & & & 0 \\
\hline 65 & & & & & & & & & & & 0,800 & 0,100 & & & & 0,100 \\
\hline 70 & & & & & & & & & & & & 0,400 & 0,400 & & & 0,200 \\
\hline 75 & & & & & & & & & & & & & 1,000 & & & 0 \\
\hline$\geq 80$ & & & & & & & & & & & & & & 0,667 & 0,267 & 0,067 \\
\hline \multicolumn{17}{|l|}{ Próx } \\
\hline M & & & & & & & & & & & & & & & & \\
\hline
\end{tabular}

CD - classes diamétricas; $R$ - recrutamento; $M$ - mortalidade.

Tabela 3 - Matriz de transição probabilística $\left(\mathrm{p}_{\mathrm{ij}}\right)$ dois passos adiante (até 2001).

\begin{tabular}{|c|c|c|c|c|c|c|c|c|c|c|c|c|c|c|c|c|}
\hline CD & $\mathrm{R}$ & 20 & 25 & 30 & 35 & 40 & 45 & 50 & 55 & 60 & 65 & 70 & 75 & $\geq 80$ & Próx. & $M$ \\
\hline $\mathrm{R}$ & & 0,742 & 0,146 & 0,028 & 0,003 & & & & & & & & & & & 0,082 \\
\hline 20 & & 0,654 & 0,177 & 0,013 & 0,001 & & & & & & & & & & & 0,075 \\
\hline 25 & & & 0,593 & 0,184 & 0,024 & 0,001 & & & & & & & & & & 0,096 \\
\hline 30 & & & & 0,598 & 0,146 & 0,017 & & & & & & & & & & 0,106 \\
\hline 35 & & & & & 0,631 & 0,289 & 0,031 & & & & & & & & & 0,022 \\
\hline 40 & & & & & & 0,682 & 0,277 & 0,020 & & & & & & & & 0,020 \\
\hline 45 & & & & & & & 0,592 & 0,183 & 0,016 & & & & & & & 0,094 \\
\hline 50 & & & & & & & & 0,669 & 0,225 & 0,006 & & & & & & 0,054 \\
\hline 55 & & & & & & & & & 0,694 & 0,076 & & & & & & 0,104 \\
\hline 60 & & & & & & & & & & 1,000 & & & & & & 0 \\
\hline 65 & & & & & & & & & & & 0,640 & 0,120 & 0,040 & & & 0,100 \\
\hline 70 & & & & & & & & & & & & 0,160 & 0,560 & & & 0,080 \\
\hline 75 & & & & & & & & & & & & & 1,000 & & & 0 \\
\hline$\geq 80$ & & & & & & & & & & & & & & 0,444 & 0,178 & 0,044 \\
\hline Próx. & & & & & & & & & & & & & & & & \\
\hline M & & & & & & & & & & & & & & & & \\
\hline
\end{tabular}




\section{AVALIAÇÃO DAS PROJEÇÕES PARA A DISTRIBUIÇÃO DIAMÉTRICA}

A Tabela 4 apresenta o produto final da Cadeia de Markov. É a projeção da distribuição de freqüência dos diferentes estados para um período seguinte de mesmo intervalo de tempo.

A projeção para 2001, com base na transição probabilística de 1999 e a ocasião imediatamente anterior (1997), indica que a taxa de mortalidade será de 44 árvores, sendo, portanto quase igual à observada em 1999 (49 árvores). A projeção do número total de árvores para as cinco parcelas permanentes, foi de 558, sem repetir a taxa de recrutamento, quando repetida essa taxa, o número de árvores será de 594 , portanto, próximo do observado em 1999 (598 árvores).

$\mathrm{Na}$ Tabela 4 , o teste $\chi^{2}$ mostra que não existe diferença significativa entre as freqüências observadas e projetadas na distribuição do número de árvores em cada classe diamétrica para o ano de $2001\left(\chi_{\text {Calculado }}^{2}=16,74\right)$. Em todas as classes diamétricas os valores do número de árvores projetadas aproximaram-se satisfatoriamente dos valores do número de árvores observadas, e que apenas nas classes 45-50 e 50-55 $\mathrm{cm}$, houve uma sutil superestimativa desses valores.

Dessa forma pode-se dizer que as projeçōes feitas para o ano 2001 foram satisfatórias. Logo, a Cadeia de Markov prova ser um bom modelo para fazer a prognose da floresta estudada.

\section{PROJEÇ̃̃O PARA 0 ANO 2005}

Depois de comprovada a qualidade das projeçōes feitas com a matriz de transição usando as medições do tempo $t$ (1997) e $t+1$ (1999) para obter uma projeção para o tempo $t+$ 2 (2001), dois passos à frente, foram feitas projeções para 2005 $(t+2)$, quatro anos à frente, onde a matriz $\mathrm{p}_{\mathrm{ij}}$, de um estado para o outro usada na Cadeia de Markov foi montada com base nas medições realizadas nas cinco parcelas permanentes estudadas, em duas ocasiōes distintas, 1997 (tempo $t$ ) e 2001 (tempo $t+1)$.

A Tabela 5 apresenta a freqüência de transição, em números absolutos, de um estado para outro, ocorrida durante o período considerado para a projeção, usando todas as observações das cinco parcelas.

Nas observações de 2001, o estado R, apresentou 63 indivíduos, e o estado $\mathrm{M}$, apresentou 66 indivíduos, ou seja, o número de árvores que entraram no sistema por meio do recrutamento foi bem próximo do número de árvores que saíram do sistema por meio da mortalidade. Em 1997, ocasião da primeira medição, foi observado um total de árvores com DAP $\geq 20 \mathrm{~cm}$ de 611 nas cinco parcelas permanentes, 674 - 63 (árvores recrutas), e o total de árvores observadas em 2001, ocasião da segunda medição, foi de 608, 674 - 66 (árvores mortas).
Tabela 4 - Freqüência observada (F.0.) em 1997, 1999 e 2001 e freqüência projetada (F.P.) para 2001 do número de árvores vivas, mortas e valores do teste Qui-quadrado $\left(\chi^{2}\right)$ calculado por classe de diâmetro.

\begin{tabular}{|c|c|c|c|c|c|c|c|}
\hline \multirow{2}{*}{$\begin{array}{l}\text { Classe } \\
\text { Diamétrica }\end{array}$} & \multicolumn{4}{|c|}{ Freqüência do número de árvores } & \multirow{2}{*}{$\chi^{2}$} & \multicolumn{2}{|c|}{ Mortalidade } \\
\hline & $\begin{array}{c}\text { F.0. } \\
1997\end{array}$ & $\begin{array}{c}\text { F.0. } \\
1999 \\
\end{array}$ & $\begin{array}{c}\text { F.0. } \\
2001\end{array}$ & $\begin{array}{c}\text { F.P. } \\
2001\end{array}$ & & $\begin{array}{c}\text { F.0. } \\
1999 \\
\end{array}$ & $\begin{array}{c}\text { F.P. } \\
2001\end{array}$ \\
\hline 20 & 178 & 177 & 174 & 176 & 0,03 & 14 & 13 \\
\hline 25 & 126 & 119 & 121 & 113 & 0,50 & 13 & 12 \\
\hline 30 & 75 & 74 & 88 & 72 & 3,37 & 10 & 8 \\
\hline 35 & 73 & 66 & 67 & 60 & 0,75 & 2 & 2 \\
\hline 40 & 46 & 51 & 53 & 54 & 0,01 & 0 & 1 \\
\hline 45 & 26 & 28 & 25 & 30 & 0,96 & 3 & 2 \\
\hline 50 & 22 & 21 & 21 & 32 & 3,64 & 1 & 1 \\
\hline 55 & 24 & 23 & 22 & 22 & 0 & 3 & 3 \\
\hline 60 & 10 & 11 & 10 & 12 & 0,32 & 0 & 0 \\
\hline 65 & 10 & 8 & 6 & 6 & 0,03 & 1 & 1 \\
\hline 70 & 5 & 3 & 5 & 2 & 4,50 & 1 & 0 \\
\hline 75 & 1 & 3 & 3 & 4 & 0,34 & 0 & 0 \\
\hline$\geq 80$ & 15 & 10 & 8 & 7 & 0,27 & 1 & 1 \\
\hline Próxima & & 4 & 5 & 3 & 2,03 & & 0 \\
\hline Total & 611 & 598 & 608 & $594^{*}$ & 16,74 & 49 & 44 \\
\hline
\end{tabular}

* Incluindo a taxa de recrutamento.

A Tabela 6 apresenta a matriz $\mathrm{p}_{\mathrm{ij}}$, onde cada probabilidade foi calculada pela divisão da freqüência do estado pela soma total da linha na matriz - freqüência total da classe na primeira ocasião (Tabela 5).

A probabilidade associada à permanência de árvores da classe 20-25 cm no período de 1997-2001, é dada por $125 / 178=0,702$, isto significa dizer que, $70,2 \%$ do total de árvores dessa classe não apresentaram crescimento em diâmetro suficiente para uma mudança de classe no período considerado. De forma análoga, a mortalidade na mesma classe é dada por $18 / 178=0,101$, pois $10,1 \%$ das árvores dessa classe morreram no período considerado para a projeção (1997-2001).

Em termos de mortalidade, semelhante aos resultados encontrados nas projeçôes para o ano de 2001, não há uma tendência, tendo em vista que as árvores das classes diamétricas menores têm as mesmas probabilidades de morrerem (saírem do sistema) que as árvores de maiores diâmetros. Já no recrutamento, ao contrário, $77,8 \%$, ocorrem na primeira classe diamétrica, $20-25 \mathrm{~cm}$. 
ACTA

AMAZONICA

Tabela 5 - Transição do número de árvores em cada classe diamétrica em DAP (cm), durante o período de 1997 - 2001.

\begin{tabular}{|c|c|c|c|c|c|c|c|c|c|c|c|c|c|c|c|c|c|}
\hline$C D$ & $\mathrm{R}$ & 20 & 25 & 30 & 35 & 40 & 45 & 50 & 55 & 60 & 65 & 70 & 75 & $\geq 80$ & Próx. & M & 1997 \\
\hline $\mathrm{R}$ & & 49 & 5 & 7 & 2 & & & & & & & & & & & & 63 \\
\hline 20 & & 125 & 33 & 2 & & & & & & & & & & & & 18 & 178 \\
\hline 25 & & & 83 & 24 & 3 & & & & & & & & & & & 16 & 126 \\
\hline 30 & & & & 55 & 10 & & & & & & & & & & & 10 & 75 \\
\hline 35 & & & & & 52 & 16 & & & & & & & & & & 5 & 73 \\
\hline 40 & & & & & & 37 & 8 & & & & & & & & & 1 & 46 \\
\hline 45 & & & & & & & 17 & 6 & & & & & & & & 3 & 26 \\
\hline 50 & & & & & & & & 15 & 5 & & & & & & & 2 & 22 \\
\hline 55 & & & & & & & & & 17 & 2 & & & & & & 5 & 24 \\
\hline 60 & & & & & & & & & & 8 & 2 & & & & & 0 & 10 \\
\hline 65 & & & & & & & & & & & 4 & 3 & & & & 3 & 10 \\
\hline 70 & & & & & & & & & & & & 2 & 2 & & & 1 & 5 \\
\hline 75 & & & & & & & & & & & & & 1 & & & 0 & 1 \\
\hline$\geq 80$ & & & & & & & & & & & & & & 8 & 5 & 2 & 15 \\
\hline \multicolumn{18}{|l|}{ Próx. } \\
\hline \multicolumn{18}{|l|}{$M$} \\
\hline 2001 & & 174 & 121 & 88 & 67 & 53 & 25 & 21 & 22 & 10 & 6 & 5 & 3 & 8 & 5 & 66 & 674 \\
\hline
\end{tabular}

$\mathrm{CD}$ - classes diamétricas; $\mathrm{R}$ - recrutamento; $\mathrm{M}$ - mortalidade.

Tabela 6 - Matriz de transição probabilística de um estado (i) para outro (j), durante um período de quatro anos após a exploração (1997-2001).

\begin{tabular}{|c|c|c|c|c|c|c|c|c|c|c|c|c|c|c|c|}
\hline CD & $\mathrm{R}$ & 20 & 25 & 30 & 35 & 40 & 45 & 50 & 55 & 60 & 65 & 70 & 75 & $\geq 80$ & Próx. \\
\hline $\mathrm{R}$ & & 0,778 & 0,079 & 0,111 & 0,032 & & & & & & & & & & \\
\hline 20 & & 0,702 & 0,185 & 0,011 & & & & & & & & & & & \\
\hline 25 & & & 0,659 & 0,190 & 0,024 & & & & & & & & & & \\
\hline 30 & & & & 0,733 & 0,133 & & & & & & & & & & \\
\hline 35 & & & & & 0,712 & 0,219 & & & & & & & & & \\
\hline 40 & & & & & & 0,804 & 0,174 & & & & & & & & \\
\hline 45 & & & & & & & 0,654 & 0,231 & & & & & & & \\
\hline 50 & & & & & & & & 0,682 & 0,227 & & & & & & \\
\hline 55 & & & & & & & & & 0,708 & 0,083 & & & & & \\
\hline 60 & & & & & & & & & & 0,800 & 0,200 & & & & \\
\hline 65 & & & & & & & & & & & 0,400 & 0,300 & & & \\
\hline 70 & & & & & & & & & & & & 0,400 & 0,400 & & \\
\hline 75 & & & & & & & & & & & & & 1,000 & & \\
\hline$\geq 80$ & & & & & & & & & & & & & & 0,533 & 0,333 \\
\hline Próx & & & & & & & & & & & & & & & \\
\hline M & & & & & & & & & & & & & & & \\
\hline
\end{tabular}


A Tabela 7 apresenta os resultados da projeção da transição para um período adiante (2005) considerando a matriz $p_{i j}$ (Tabela 6) que foi calculada usando as probabilidades da matriz $\mathrm{p}_{\mathrm{ij}}{ }^{2}$.

O produto final da Cadeia de Markov é a projeção da distribuição de freqüência dos diferentes estados, recrutamento (estado 1), mortalidade (estado 16) e as classes diamétricas (estados 2 a 15), para um período seguinte de mesmo intervalo de tempo (Tabela 8).

A projeção para 2005, com base na transição probabilística de 2001 e a ocasiáo imediatamente anterior (1997) indica que a taxa de mortalidade será bem próxima a anterior $(2005=58$ e $2001=66$ ), e se repetida a taxa de recrutamento (63 árvores) em 2005, o número total de árvores será de 625 , um pouco maior que o número observado em 2001.

Em florestas submetidas à exploração florestal e monitoradas por meio de parcelas permanentes, uma estabilização do comportamento em termos de recrutamento e mortalidade deve ser aguardada para a previsão utilizando a Cadeia de Markov, pois logo após a abertura da clareira, decorrente da exploração, o processo dinâmico desse ambiente tornase acelerado nos primeiros anos, principalmente, devido a mudanças bruscas em termos de quantidade e qualidade de luz, espaço, processo de regeneração de clareiras, competição e mortalidade causadas por danos decorrentes da exploração. Portanto, um período de 5 a 10 anos, quando a floresta
Tabela 8 - Freqüência observada (F.0.) em 1997 e 2001 e freqüência projetada (F.P.) para 2005 do número de árvores vivas e mortas, por classe de diâmetro.

\begin{tabular}{|c|c|c|c|c|c|}
\hline \multirow{2}{*}{$\begin{array}{l}\text { Classe } \\
\text { Diamétrica }\end{array}$} & \multicolumn{3}{|c|}{ Freqüência do número de árvores } & \multicolumn{2}{|l|}{ Mortalidade } \\
\hline & F.0. 1997 & F.0. 2001 & F.P. 2005 & F.0. 2001 & F.P. 2005 \\
\hline 20 & 178 & 174 & 122 & 18 & 17 \\
\hline 25 & 126 & 121 & 112 & 16 & 14 \\
\hline 30 & 75 & 88 & 90 & 10 & 8 \\
\hline 35 & 73 & 67 & 62 & 5 & 4 \\
\hline 40 & 46 & 53 & 57 & 1 & 2 \\
\hline 45 & 26 & 25 & 26 & 3 & 3 \\
\hline 50 & 22 & 21 & 43 & 2 & 2 \\
\hline 55 & 24 & 22 & 20 & 5 & 4 \\
\hline 60 & 10 & 10 & 10 & 0 & 1 \\
\hline 65 & 10 & 6 & 4 & 3 & 2 \\
\hline 70 & 5 & 5 & 4 & 1 & 0 \\
\hline 75 & 1 & 3 & 5 & 0 & 0 \\
\hline$\geq 80$ & 15 & 8 & 4 & 2 & 1 \\
\hline Próxima & & 5 & 3 & & 0 \\
\hline Total & 611 & 608 & $562^{*}$ & 66 & 58 \\
\hline
\end{tabular}

* sem incluir a taxa de recrutamento.

Tabela 7 - Matriz de transição probabilística quatro passos adiante (até 2005).

\begin{tabular}{|c|c|c|c|c|c|c|c|c|c|c|c|c|c|c|c|c|}
\hline$C D$ & $\mathrm{R}$ & 20 & 25 & 30 & 35 & 40 & 45 & 50 & 55 & 60 & 65 & 70 & 75 & $\geq 80$ & Próx. & $M$ \\
\hline $\mathrm{R}$ & & 0,546 & 0,196 & 0,105 & 0,039 & 0,007 & & & & & & & & & & 0,106 \\
\hline 20 & & 0,493 & 0,252 & 0,051 & 0,006 & & & & & & & & & & & 0,096 \\
\hline 25 & & & 0,434 & 0,265 & 0,058 & 0,005 & & & & & & & & & & 0,111 \\
\hline 30 & & & & 0,538 & 0,193 & 0,029 & & & & & & & & & & 0,107 \\
\hline 35 & & & & & 0,507 & 0,332 & 0,038 & & & & & & & & & 0,054 \\
\hline 40 & & & & & & 0,647 & 0,254 & 0,040 & & & & & & & & 0,038 \\
\hline 45 & & & & & & & 0,428 & 0,308 & 0,052 & & & & & & & 0,096 \\
\hline 50 & & & & & & & & 0,465 & 0,316 & 0,019 & & & & & & 0,109 \\
\hline 55 & & & & & & & & & 0,502 & 0,126 & 0,017 & & & & & 0,148 \\
\hline 60 & & & & & & & & & & 0,640 & 0,240 & 0,060 & & & & 0,060 \\
\hline 65 & & & & & & & & & & & 0,160 & 0,240 & 0,120 & & & 0,180 \\
\hline 70 & & & & & & & & & & & & 0,160 & 0,560 & & & 0,080 \\
\hline 75 & & & & & & & & & & & & & 1,000 & & & 0 \\
\hline$\geq 80$ & & & & & & & & & & & & & & 0,284 & 0,178 & 0,071 \\
\hline Próx. & & & & & & & & & & & & & & & & \\
\hline
\end{tabular}

CD - classes diamétricas; R - recrutamento; $\mathrm{M}$ - mortalidade. 
começa a retornar as características semelhantes às que tinha antes da exploração, deve ser aguardado para projeçóes mais confiáveis.

A Cadeia de Markov ou Matriz de Transição é um eficiente instrumento para projetar a dinâmica da floresta natural (Scolforo, 1998; Schneider \& Finger, 2000; Rocha, 2001; Teixeira et al., 2007) contribuindo para o planejamento e ações de conservação em curto prazo das atividades que utilizam os recursos florestais.

\section{CONCLUSÃO}

O comportamento dinâmico observado na floresta indica que não existe um padrão definido para a dinâmica nas classes diamétricas em termos de mortalidade ou crescimento, apresentando um comportamento aleatório ou probabilístico, justificando assim, a utilização de um modelo estocástico em florestas tropicais, como a Cadeia de Markov.

Nas cinco parcelas estudadas, o teste $\chi^{2}$ revelou que não houve diferença significativa entre freqüências projetadas e observadas para a distribuição do número de árvores em cada classe diamétrica. Portanto, a Cadeia de Markov pode ser considerada como uma ferramenta potencial para a projeção do comportamento futuro de florestas tropicais na Amazônia em termos de distribuição de freqüência das classes diamétricas, recrutamento e mortalidade, reproduzindo, com sensata aceitação, o processo dinâmico das árvores nas classes diamétricas.

Essas projeções podem ser de grande contribuição para o manejo de florestas tropicais, auxiliando na determinação do ciclo de corte ou mostrando as tendências que a floresta de hoje apresentará em um futuro próximo.

\section{AGRADECIMENTOS}

Os autores agradecem a Empresa Brasileira de Pesquisa Agropecuária - Centro de Pesquisa Agroflorestal do Acre pelo fomento à pesquisa, a Coordenação de Aperfeiçoamento de Pessoal de Nível Superior (CAPES) pela bolsa de mestrado concedida ao primeiro autor.

\section{BIBLIOGRAFIA CITADA}

Azevedo, C.P. 1993. Predição da distribuição diamétrica de povoamentos florestais ineqüiâneos pelo emprego da matriz de transição. Dissertação de Mestrado, Universidade Federal de Viçosa, Viçosa, Minas Gerais. 118pp.

Bruner, H.D.; Moser, J.W. 1973. A Markov Chain approach to the prediction of diameter distributions in uneven-aged Forest stands. Canadian Journal of Forest Research, Ontario. 3: 409417.
Costa Neto, F.; Couto, J.; Ramalho, R.S.; Gomes, J.M. 1991. Subsídios técnicos para um plano de manejo sustentados em áreas de cerrado. Revista Árvore, 15 (3): 241-256.

Durigan, G.; Garrido, L.M.A.G.; Garrido, M.A.O. 1993. Manejo silvicultural do cerrado em Assis-SP. In: I Congresso Florestal Pan-americano e VII Congresso Florestal Brasileiro, Anais... Curitiba, 1993, S.B.S/S.B.E.F., 11: 374-377.

EMBRAPA - Empresa Brasileira de Pesquisa Agropecuária CPAF/ ACRE. 1996. Boletim Agro-meteorológico, 5: 1990-1994.

Freitas, J.V.; Higuchi, N. 1993. Projeções da distribuição diamétrica de uma floresta tropical úmida de terra firme com a utilização da cadeia de Markov. In: I Congresso Florestal Panamericano e VII Congresso Florestal Brasileiro, Anais. Curitiba, Paraná, S.B.S. /S.B.E.F., 11: 545-548.

Higuchi, N. 1987. Short-term growth of an undisturbed tropical moist forest in the Brazilian Amazon. D. Phil. Thesis, Michigan State University. Michigan, USA. 129pp.

Higuchi, N.; Vieira, G. 1990. Manejo sustentado da floresta tropical úmida de terra firme na região de Manaus - Um projeto de pesquisa do INPA. In: VI Congresso Florestal Brasileiro, Anais... Campos do Jordão, S.B.S/S.B.E.F., 11: 34-37.

Mendoza, G.A.; Setyarso, A. 1986. Transition matrix forest growth model for evaluating alternative harvesting schemes in Indonesia. Forest Ecology and Management, 15: 219-228.

Oliveira, M.V.N.d'. 2000. Sustainable forest management for small farmers in Acre state in the Brazilian Amazon. D. Phil. Thesis, Aberdeen University, Scotland, U. K. 161pp.

Pulz, F.A.; Scolforo, J.R.; Oliveira, A.D.; Mello, J.M.; Oliveira Filho, A.T. 1999. Acuracidade da predição da distribuição diamétrica de uma floresta inequiânea com a matriz de transição. Revista Cerne. 5(1): 001-014.

Rocha, R.M. 2001. Taxa de recrutamento e mortalidade da floresta de terra-firme da bacia do rio Cuieiras na regiāo de Manaus-AM. Dissertação de Mestrado, Instituto Nacional de Pesquisa da Amazônia, Fundação Universidade do Amazonas, Manaus, Amazonas. 49pp.

Sanquetta, C.R.; Ângelo, H.; Brena, D.A.; Mendes, J.B. 1996. Matriz de transição para simulação da dinâmica de florestas naturais sob diferentes intensidades de corte. Revista Ciência Florestal, 6(1): 65-78.

Sanquetta, C.R.; Arce, J.E.; Gomes, F.; Cruz, E.C. 1999. Evaluación y simulación precoces del crescimento de rodales de Pinus taeda L. com matrices de transición. Revista de Ciências Forestales, 7:31-42

Schneider. P. R.; Finger, C. A. G. 2000. Manejo sustentado de florestas inequiâneas heterogêneas. UFSM, Santa Maria. 195pp.

Scolforo, J.R.S. 1998. Manejo florestal. Lavras: UFLA/FAEPE, $443 \mathrm{pp}$.

Scolforo, J.R.; Silva, S.T. 1993. O conceito de floresta balanceada de Meyer como opção para intervenção em cerrado senso stricto. In: I Congresso Florestal Pan-americano e VII Congresso Florestal Brasileiro, Anais... Curitiba, S.B.S/S.B.E.F., 11: 378-381. 
SEPLAN - Secretaria de Estado de Planejamento e Coordenação. 2000. Zoneamento Ecológico-Econômico do Estado do Acre. Recursos Naturais e Meio Ambiente. Rio Branco, Acre. Vol. 1. 116pp.

Silva, J.N.M. 1989. The behavior of the tropical rain forest of the Brazilian Amazon after logging. D. Phil. Thesis. University of Oxford. 302pp.

Solomon, D.S.; Hosmer, R.A.; Hayslett, H.T. 1986. A two-stage matrix model for predicting of forest stands in the northeast. Canadian Journal of Forest Research, 16: 521-528.

Teixeira, L.M.; Chambers, J.Q.; Silva, A.R.; Lima, A.J.N.; Carneiro, V.M.C.; Santos, J. dos; Higuchi, N. 2007. Projeção da dinâmica da floresta natural de Terra-firme, região de Manaus-AM, com o uso da cadeia de transição probabilística de Markov. Acta Amazônica, 37 (3):377-384.
Vasconcelos, S.S. 2003. Dinâmica de uma floresta explorada seletivamente no Projeto de Colonização Pedro Peixoto na Amazônia Ocidental. Dissertação de Mestrado, Instituto Nacional de Pesquisa da Amazônia, Fundação Universidade do Amazonas, Manaus, Amazonas. 71pp.

Recebido em 04/10/2007

Aceito em 16/06/2008 